\title{
Lire le travail dans les cartes
}

Learning the job with playing cards

\section{Roger Cornu}

\section{OpenEdition}

Journals

Édition électronique

URL : http://journals.openedition.org/itti/892

DOI : 10.4000/itti.892

\section{Éditeur}

Université de Poitiers

\section{Référence électronique}

Roger Cornu, "Lire le travail dans les cartes », Images du travail, travail des images [En ligne], 5| 2018, mis en ligne le 01 février 2018, consulté le 14 avril 2021. URL : http://journals.openedition.org/itti/892 ; DOI : https://doi.org/10.4000/itti.892

Ce document a été généré automatiquement le 14 avril 2021.

Images du travail, travail des images 


\title{
Lire le travail dans les cartes
}

\author{
Learning the job with playing cards
}

\section{Roger Cornu}

1 L'acculturation au monde du travail passe par un ensemble d'images, de représentations du travail auxquelles on est confronté depuis la plus tendre enfance et tout au long de la vie. Tous les secteurs d'activité sociale participent à la production de ces représentations de façon plus ou moins complexe. Ils fournissent aux sociologues des corpus d'informations dont certains sont peu ou pas utilisés, soit qu'on les considère comme tout à fait secondaires, soit qu'on ne sache pas comment les utiliser. C'est le cas notamment des jeux de cartes qui véhiculent de nombreuses représentations du travail. L'alliance du jeu et du travail apparait paradoxale car rien, dans la morale dominante, n'est plus immédiatement contradictoire que ces deux termes. On ne peut que se demander comment le jeu de cartes qui fut officiellement ou implicitement condamné comme source de déchéance, de fainéantise et de ruine, a pu diffuser des représentations du travail qui, lui, fut, du moins pour le peuple, hautement valorisé. La représentation du travail ne peut toutefois être comprise que si l'on n'oublie pas que l'on a affaire à un jeu codifié dans sa structure et dans son utilisation, et que les producteurs de jeu ne peuvent pas trop s'éloigner de ce code s'ils veulent que le jeu soit reconnu comme tel. C'est aussi un ensemble d'images et toute représentation doit combiner la tradition iconographique des jeux et les tendances iconographiques de l'époque. L'analyse d'un corpus de jeux de cartes permet de préciser ces questions et de s'interroger sur la complexité d'une analyse de ce type de représentations du travail.

\section{Jeux de cartes et règles du jeu}

2 Peu d'ensembles iconographiques resteront aussi stéréotypés à travers les âges. Plusieurs raisons sont à la base de ces traits permanents. Nous ne pouvons ici n'en énumérer que quelques-unes :

- Lorsque les jeux de cartes apparaissent à la fin du XlVe siècle, l'image se fabrique et se lit comme les sculptures des porches ou les vitraux des cathédrales. Le jeu est ainsi un livre de lecture. 
- S'écarter du code, c'est perdre le sens. Les cartes vont être utilisées pour des jeux multiples à base d'argent. Le jeu implique une règle stricte connue de tous et la règle ne peut se construire qu'autour d'une structure stable du jeu de cartes.

- La signification du code original de l'imagerie ayant été perdue dès le début du XVIIe siècle, le jeu devient mystère et sera utilisé pour découvrir son destin. L'ensemble iconographique devient représentation de l'univers et doit rester aussi stable que lui. L'intervention de l'État, contrôlant la production des jeux de cartes et imposant ses taxes, va s'accompagner, pour la France, de la définition d'un portrait officiel.

- Enfin, même avec la libéralisation de la fabrication des jeux, les producteurs ne pourront guère s'écarter de la structure traditionnelle et ce pour deux raisons principales : un jeu de cartes est support d'un ensemble de règles de jeux multiples qui referme la structure du jeu de cartes sur sa forme originale; le joueur doit repérer rapidement les valeurs des cartes et donnera sa préférence au jeu le plus souvent utilisé, le plus stéréotypé.

\section{La structure du jeu}

3 Retenons ici le jeu de tarot qui réunit les caractéristiques de tous les autres jeux. Il se compose d'abord de trois types de cartes : les cartes numérotées (de 1 à 10); les cartes habillées combinant les figures des jeux italiens ou espagnols et des jeux français (rois, dames, cavaliers, valets) ; les cartes armoriées qui lui sont propres et qui racontent une ou des histoires.

4 Les cartes numérotées et les cartes habillées se divisent en deux couleurs (rouge et noir) pour le jeu français classique et en quatre emblèmes pour tous les jeux, qu'ils portent les emblèmes français (pique, cœur, carreau, trèfle), italo-espagnols (épée, coupe, denier, bâton) ou allemands (gland, cœur, grelot, feuille).

5 La structure en quatre emblèmes contraindra les cartiers qui veulent innover à donner la préférence à des représentations de phénomènes qui se structurent autour du nombre 4. Ce sera les quatre types d'instruments de musique (bois, cuivres, cordes, percussions), ou les quatre saisons, ou quatre pays pris dans une alliance ou dans un conflit, etc.

6 De même la présence, dans les jeux courants, de trois cartes habillées (ou figures), par couleur, sera une nouvelle contrainte d'autant plus que ces cartes sont socialement hiérarchisées. Ainsi, Saint Simon, lors de la Révolution Française remplacera-t-il les rois par des génies (de la guerre, de la paix, des arts, du commerce), les dames par des libertés (de cultes, de mariage, de la presse, des professions), les valets par des égalités (de devoirs, de droits, de rangs, de couleurs). Un autre jeu de la période révolutionnaire produit à Toulouse remplace les rois par les quatre éléments, les dames par les quatre saisons, et les valets par quatre cultivateurs.

\section{La règle du jeu}

7 Le nombre de façons de jouer en utilisant toujours le même modèle de jeu ira en augmentant au cour des siècles. Avant même de fixer le déroulement du jeu, les règles modifient éventuellement la valeur et l'ordre des cartes. Ces modifications peuvent toutefois renvoyer soit à la structure ancienne du jeu de cartes, soit au transfert d'une façon de jouer d'un type de jeux à un autre. Deux exemples permettent d'éclairer ce 
point. Les jeux italiens ou espagnols comportent des cartes numérotées de 1 à 7 (jeu de 40 cartes) ou de 1 à 9 (jeu de 48 cartes). Dans les deux cas, le 10 est une carte absente. Si l'on considère la règle du jeu de belote et l'ordre des cartes à l'atout, le 34 se compose du valet et du 9, c'est-à-dire des deux cartes connexes qui articulent les cartes numérotées et les cartes habillées dans le jeu de 48 cartes. On assiste là à une double transformation : changement de hiérarchie et transfert d'une connexité de cartes d'un type de jeu à un autre où cette connexité n'existe pas : le jeu de 32 cartes.

De même dans le jeu de belote et le jeu de manille, as et 10 se côtoient mais dans un rapport inverse quant à l'ordre des valeurs. Ces deux cartes portent un nom, les brisques, dans de multiples jeux et renvoient à deux cartes qui n'avaient pas de valeur dans les anciens jeux, l'une portant le nom du fabricant (l'as) et l'autre portant un drapeau et des armoiries. Ces brisques eurent une telle importance et manifestèrent si précisément le lieu où l'on joue et le statut social du joueur, que les sous-officiers de l'armée furent appeler les « briscards ».

9 Les règles du jeu valorisent par ailleurs, différents types de regroupements, en lignes ou en colonnes, diraient les statisticiens d'aujourd'hui. En ligne, c'est-à-dire ayant même valeur, par deux comme au jeu de bataille, par trois dans le cas du brelan, ou quatre pour le carré. En colonne, par trois, dans le cas des suites, jusqu'à l'ensemble des cartes d'un même emblème (une même couleur dit-on couramment). Enfin, la règle peut aussi favoriser les mariages : la dame de "ceci" avec le "valet" ou le "roi" de cela permettant ainsi d'allier à la fois les emblèmes et les rangs.

Dans le déroulement du jeu enfin, les cartes se présentent dans un ordre conjoncturel (jeu battu), dissimulant l'ordre structurel. Elles sont réparties entre plusieurs mains qui comportent leur propre logique interne et se manifestent dans un ordre nouveau à travers les plis, ordre déterminé à la fois par la structure du jeu, et la maîtrise que les joueurs ont du déroulement du jeu.

11 Il est difficile de traiter des représentations sociales présentes dans l'iconographie sans attirer l'attention sur ces filtres que le fabricant de cartes doit essayer de prendre en compte, auxquels le joueur se trouve confronté et qui influent sur l'intériorisation non réfléchie de cet ensemble de représentations.

\section{Techniques de fabrication et utilisations}

On peut dire que, jusqu'à la Révolution française, les jeux sont destinés pour l'essentiel à la noblesse ou à la haute bourgeoisie. Les premiers jeux de cartes sont peints à la main à la demande d'un propriétaire précis. On connaît ainsi le jeu de Charles Vll ou les tarots des Visconti. Produit de luxe, le jeu aura une clientèle limitée. Seconde forme d'impression qui permet d'étendre la clientèle, la gravure sur bois, technique comparable à celles utilisées par l'imagerie populaire. Les cartes pourront éventuellement combiner une forme de gravure à une coloration à la main. Jusqu'à la Révolution française, le jeu sous sa forme neuve et complète restera l'apanage des classes dominantes. Les jeux défraîchis ou incomplets passeront dans les milieux populaires. Incomplets, cela est fréquent car les nobles utilisent les cartes à jouer comme cartes de visite. Les cartiers sont la plupart du temps simultanément producteurs d'images populaires. Petits artisans, peu équipés, ils se contentent de 
reproduire ou d'adapter les modèles existants. Selon l'époque et suivant la mode, il suffira de modifier le vêtement.

13 Ce n'est qu'à la fin du XVllle siècle que la découverte de la lithographie, puis vers 1840 la mise au point de la chromolithographie, permettent la fabrication en série des jeux de cartes, les rendant accessibles à un plus grand public mais renforçant leur caractère stéréotypé. Même s'ils n'atteignent pas encore les milieux populaires, les jeux se diffuseront dans les classes moyennes abandonnant leur caractère de jeu d'argent pour devenir un jeu familial. Ce n'est qu'après 1865, avec l'apparition de la machine lithographique, puis au début du XXe siècle avec le développement de l'offset que le jeu de cartes deviendra un jeu vraiment populaire. En même temps, les nouvelles techniques conduiront à standardiser la fabrication des jeux au détriment de l'invention iconographique. La connaissance de cet aspect de l'histoire des jeux est essentiel si l'on veut pouvoir interpréter la signification des représentations du travail dans les jeux de cartes.

\section{Des cris de Paris aux petits métiers}

14 Dans les premiers jeux de cartes connus, les représentations des métiers sont exceptionnelles ou n'y figurent pas en tant qu'activité de travail mais plutôt sous forme de symboles. On peut prendre comme exemple ici les jeux hollandais qui dénoncent les spéculations de Law au XVllle siècle. On y verra ainsi un peseur d'or pour montrer la valeur de l'argent en jeu, un marchand de lunettes pour montrer l'aveuglement des victimes de Law, un balayeur de rue chargé de ramasser le papier monnaie qui jonche les rues.

Présence indirecte des métiers encore à travers la tradition des grotesques. Ainsi, un jeu allemand de Nuremberg gravé sur bois représente un paysan accroupi, sur le manche de sa faux en train de déféquer dans son champ tandis que sa femme manie le râteau. Toujours en Allemagne, au début du XIXe siècle (1810), les atouts du tarot des animaux nous montrent un taureau horloger, un hibou allumeur de réverbère, etc., dans le cadre d'un monde à l'envers que l'on retrouvera en France chez le dessinateur Grandville. 


\section{LE RÉMOULEUR}

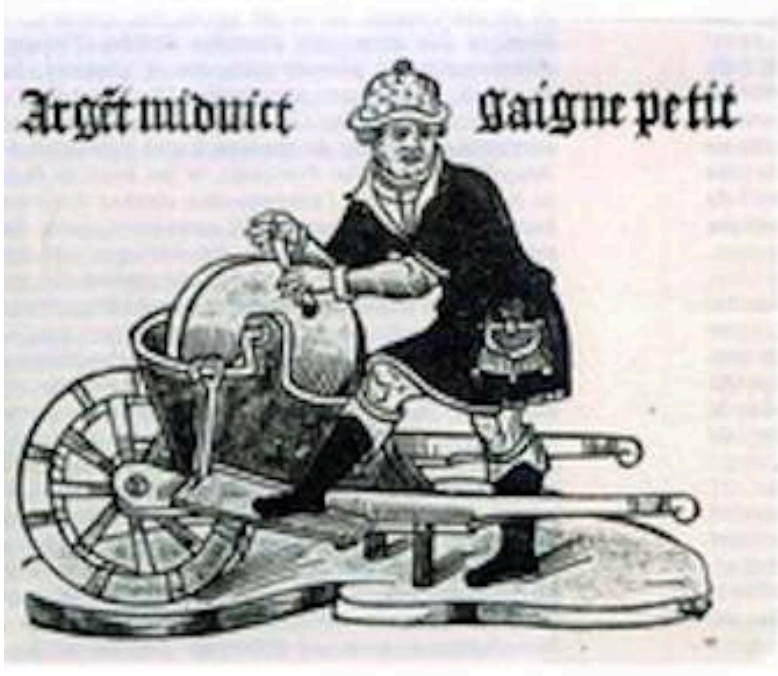

Vers 1500

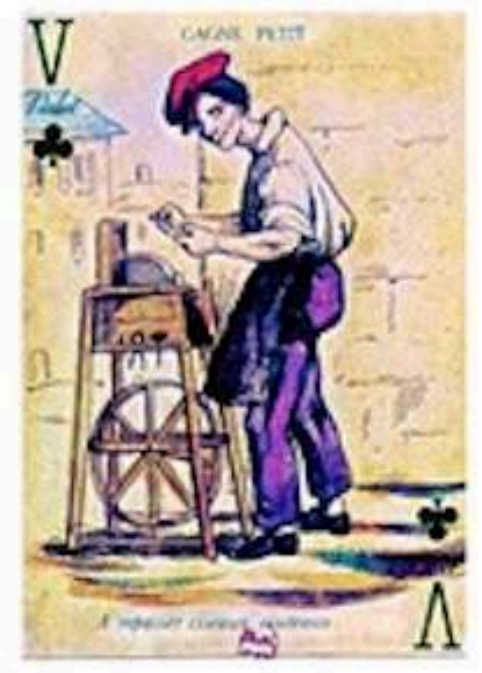

1834 l'on appelle les jeux fantaisies. Caractéristique de ce point de vue, un jeu paru en France en 1834. Les cris de Paris avait déjà fait l'objet d'un jeu de l'oie en 1808, s'ancrant dans une tradition plus longue qui nous renvoie au problème de la lecture des éléments iconographiques. Dès le XVe siècle mais surtout au XVle siècle, les cris de la ville font l'objet d'un ensemble de productions artistiques : poèmes, musiques, images. Les plus anciennes iconographies connues sont datées aux environs de 1500. C'est le cri qui est ici prioritaire, l'image ne faisant que l'illustrer. Les activités regroupées sont des activités itinérantes, des petits marchands ou des petits métiers indispensables à la vie quotidienne. Ces cris de la ville (de Paris, de Londres, de Rome) représentent une production importante de l'image de colportage ou de l'estampe. La transformation de ces images en cartes à jouer est exceptionnelle. On en trouve un jeu en Allemagne au XVIIe siècle, la légende criée étant en allemand et en français ; cartes pour nobles, si l'on en croit le mode de fabrication, gravure sur cuivre et pochoir. La gravure ou la lithographie sera relayée par la photographie dès son apparition et des photographes prestigieux, tels Charles Negre sous le Second Empire, Eugène Atget à la fin du XIXe siècle. Jérôme Doucet consacrera un volume à ces cris de Paris qui sont devenus les petits métiers de Paris (les photos sont rééditées aujourd'hui sous forme de cartes postales). De la photo, la tradition investira la carte postale, les petits métiers devenant métiers pittoresques ou objet ethnographique. 


\section{LES CRIS DE PARIS}
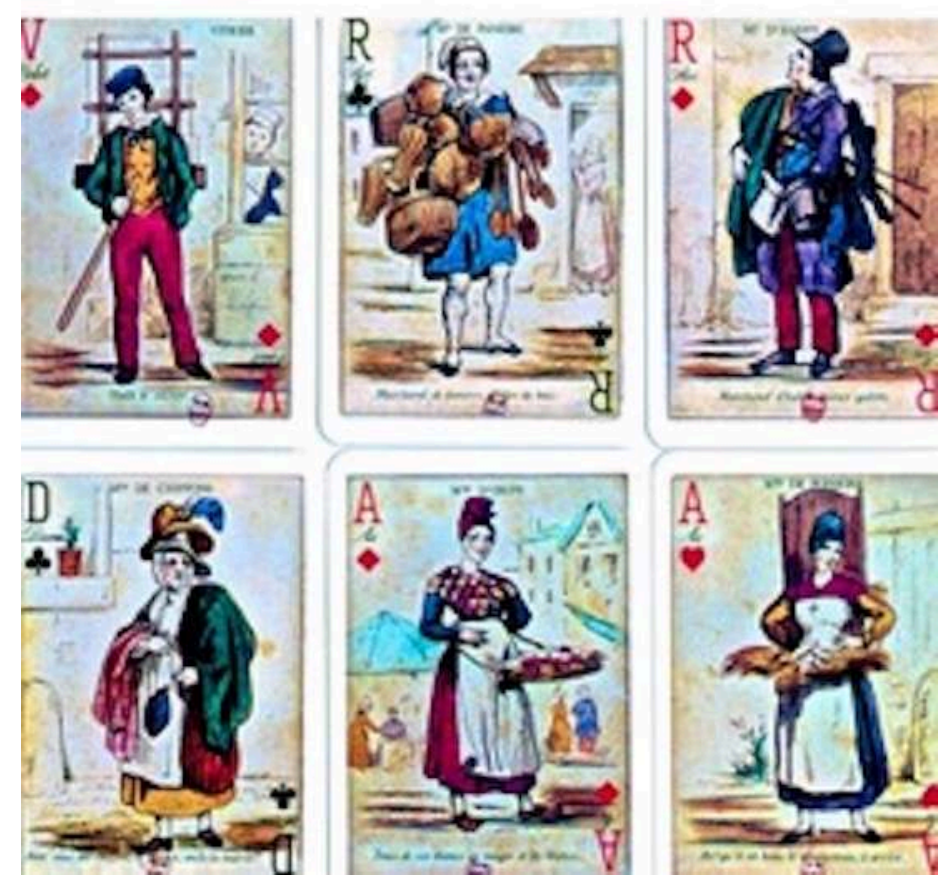

Avant d'être pris comme un document sur la société de 1834, le jeu des Cris de Paris doit être relié à toute cette tradition Si le rémouleur ou gagne-petit, lui sert d'emblème, il est déjà présent dans les représentations de 1500 et sera représenté de nombreuses fois dans les cartes postales. Il en va de même de la marchande de poissons, de la marchande d'huîtres, du marchand de gâteaux, du cordonnier. Par rapport aux différents métiers itinérants, le nombre de cartes habillables possibles est limité. Le jeu utilise l'as et les trois cartes habillées, soit 16 pour le total du jeu. De plus, la moitié des cartes, les rois et les valets sont réservés à des métiers masculins, ce qui conduit à une sélection dans les représentations traditionnelles, voire même à des changements de sexe dans les différents métiers.

Nous avons déjà noté que les conditions de production tendaient à perpétuer des personnages dont on se contentait de modifier le vêtement. On pourrait dès lors supposer que l'on peut retrouver les costumes de métiers, d'autant plus typés que ces ambulants étaient des immigrants venus des différentes provinces et contrôlant telle ou telle activité. L'image croise alors une seconde tradition, les gravures représentant les costumes nationaux, régionaux ou typique des différentes classes de la société. Il faut ajouter encore la tradition des costumes grotesques, notamment les costumes grotesques des métiers de Nicolas de Larmessin à la fin du XVlle siècle (reproduits récemment en jeu de cartes). Entre le grotesque, la tradition et la réalité, où est la réalité ?

\section{Le métier sédentaire et l'unité domestique}

Quelques cartes gravées sur bois (les autres cartes ont été perdues) d'un jeu de la seconde moitié du XVe siècle fabriqué dans le sud de l'Allemagne nous montrent des 
métiers. Ce sont les activités nécessaires à la vie d'un noble de l'époque. Il y a là le cuisinier, le barbier, celui qui apporte les chapeaux. Une jeune femme musicienne conduit toutefois à s'interroger sur les cartes manquantes. Le jeu aurait pu parfaitement représenter la journée d'un courtisan, les différentes activités apparaissant sur certaines cartes seulement comme des moments de la vie quotidienne.

Deux jeux français de la Révolution nous montrent les métiers sédentaires par excellence, ceux qui, pour les physiocrates, sont la seule et unique source de richesse, les métiers de la terre : le vendangeur, le bûcheron, le jardinier et le moissonneur. Ces cultivateurs occupent la place des valets et sont dominés dans un cas par les vertus (dames) et grands écrivains (rois), dans l'autre pour les raisons (dames) et les éléments (rois). Dans ce dernier jeu, (jeu de Lamarque à Toulouse), chaque cultivateur porte un nom quelque peu ironique. Ainsi le vendangeur se nomme «l'Altéré » et le bûcheron «| a Souche ».

Dans les costumes grotesques de Nicolas de Larmessin dont nous avons déjà parlé, on trouve aussi bien des métiers sédentaires que des métiers ambulants. Les planches gravées d'imagerie populaire de la fin du XVIIIe siècle et surtout de la première moitié du XlXe siècle présenteront ce mélange, substituant les arts et métiers au Cris de Paris. On voit alors apparaître des métiers du type épicier, tailleur, boucher, architecte, maçon, tourneur sur bois, etc. Le type d'iconographie reste toutefois proche de celle des Cris de Paris ou, dans certains cas, peut aussi faire penser à la représentation des métiers sur les vitraux des cathédrales. Ce type de production restera toutefois extérieur aux jeux de cartes, jusqu'à la fin du XlXe siècle pour la France.

Image 3

\section{Cartes divinatoires et éducatives}

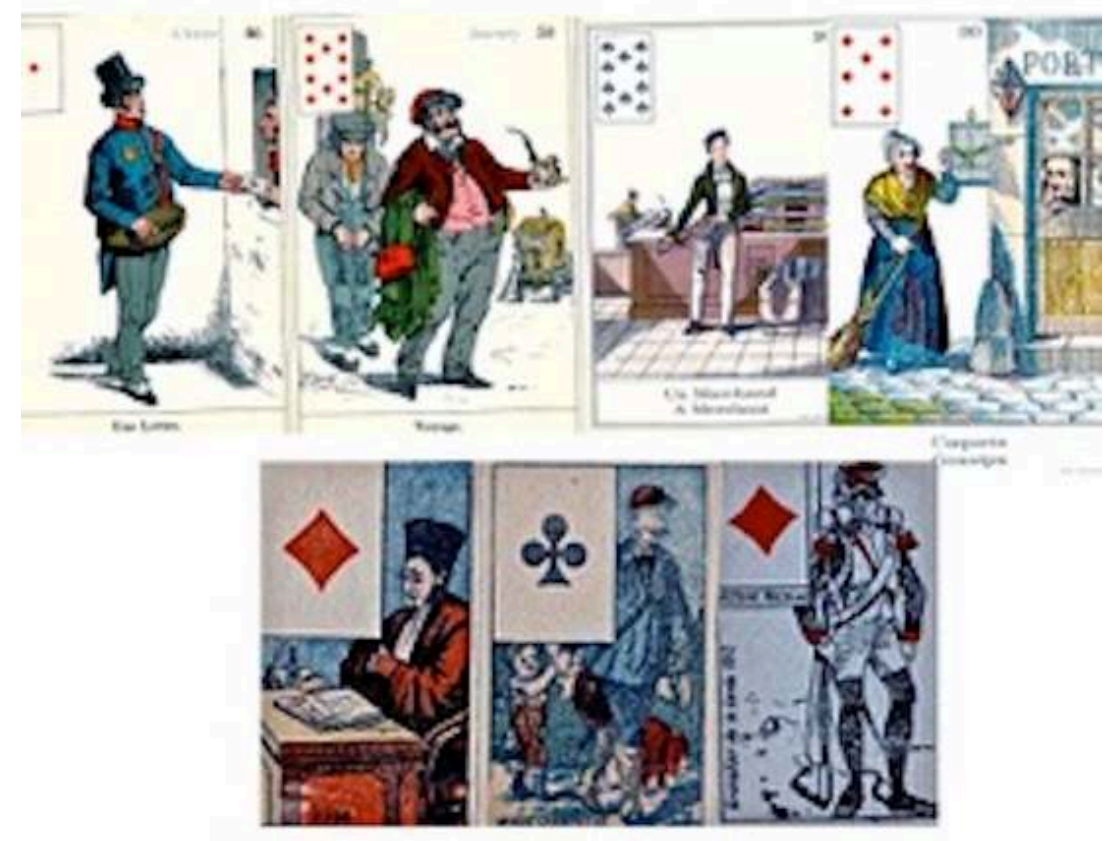

Vers 1850, apparaît en Angleterre le jeu des Joyeuses Familles. Certains auteurs considèrent que l'on est sorti des jeux car les emblèmes n'existent plus. Ce qui n'est en 
fait qu'une apparence. Les historiens de la carte à jouer s'interrogent sur l'origine des images utilisées notamment pour les arcanes majeures (ou atouts) des tarots et se demandent s'il ne s'agirait pas à l'origine d'ensembles d'images éducatives ou édifiantes. En tous cas, dès le XVIe siècle, on trouve des jeux éducatifs ou récréatifs porteurs d'emblèmes ou non destinés aux enfants et aux familles. On y apprendra aussi bien à lire que les mathématiques, la géographie, l'histoire, les institutions politiques, la religion ou les bonnes mœurs.

Image 4

\section{LES JOYEUSES FAMILLES}
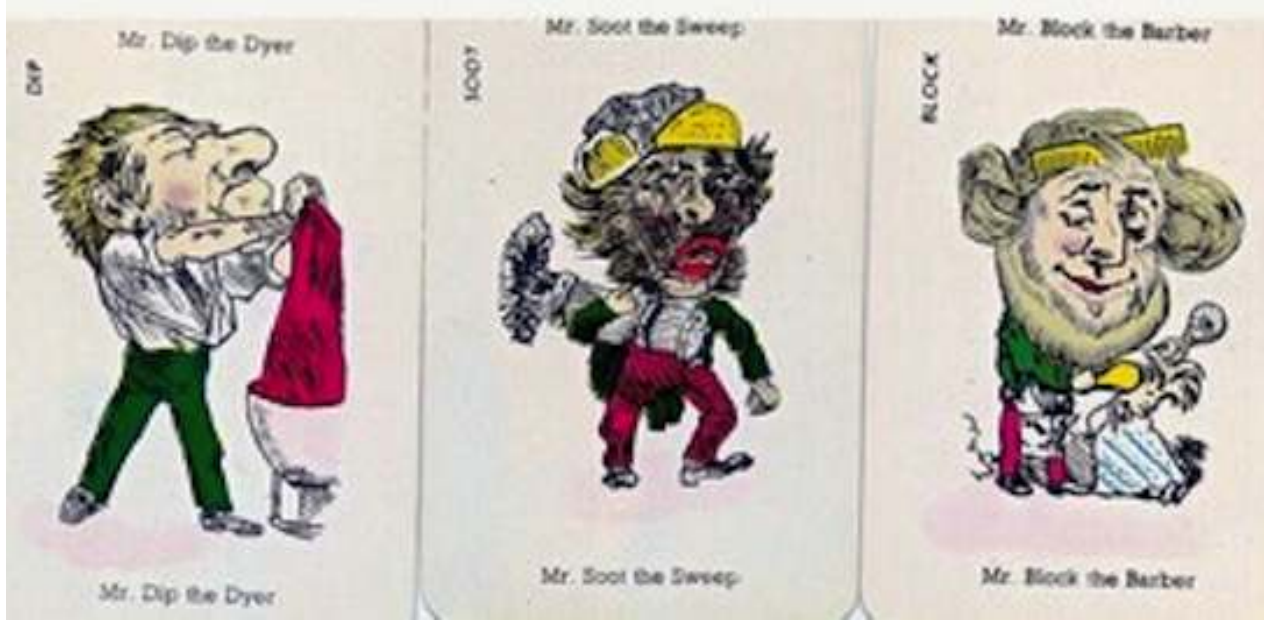

Au XlXe siècle, le jeu lui-même et son aspect récréatif rentrent dans la vie des familles des classes moyennes en perdant son aspect éducatif. C'est dans ce cadre que se place l'apparition du jeu des Joyeuses familles, où la famille est caractérisée par un métier et dont le nom fait référence à un aspect de l'activité tel « Hérisson » pour le ramoneur ou «Tonneau» pour le brasseur. Ce sont ces noms qui remplacent les emblèmes. Les illustrations des cartes se présentent sous la forme des Grotesques. La famille est composée de quatre personnes (ou quatre cartes) : le père, la mère, le garçon, la fille. Le jeu regroupe onze familles de métiers qui ont toutes à voir avec la vie quotidienne de la famille qui joue. Quatre familles renvoient à la nourriture : le boucher, le boulanger, l'épicier et le brasseur; deux à l'entretien du corps : le coiffeur et le docteur; deux pour le vêtement: le tailleur et le teinturier; enfin trois pour l'entretien de la maison: le charpentier, le ramoneur et le peintre. Chaque membre de la famille détient un emblème du métier comme si toute la famille participait au travail, dans le cadre d'une exploitation familiale et comme si le métier était héréditaire, ce qui renvoie d'ailleurs aux idées de la première moitié du XlXe siècle : stabiliser les enfants dans le métier de leurs parents, notamment en vue de la reproduction élargie du capital naissant des petites entreprises.

Lorsque le jeu apparait en France, il devient Jeu des Sept Familles (au lieu de 11) avec des familles qui ne sont plus la famille nucléaire mais une famille patriarcale à trois générations accentuant du même coup le caractère héréditaire du métier. Le premier jeu reste assez proche de son modèle anglais avec des images grotesques accompagnées 
de noms ridicules. On y retrouve trois métiers pour la nourriture: le boucher, le boulanger, l'épicier; un métier pour l'entretien du corps, le coiffeur; deux métiers de l'habillement : le tailleur et le cordonnier; enfin le peintre qui a perdu son caractère de peintre en bâtiment pour devenir artiste-peintre. Quelques soient les métiers retenus dans les autres jeux fabriqués, l'artiste-peintre restera pendant longtemps le pilier de ces jeux de Sept Familles, qu'il s'appelle Lapalette, Rapin ou Dupinceau. Après l'univers ambulant des cris de Paris, c'est le monde de la boutique et de l'atelier qui nous est présenté. Ce sont aussi les métiers dont on a besoin dans la vie quotidienne, ceux que |'on rencontre dans le quartier et pour l'artiste-peintre, c'est peut-être l'univers de ses dettes. On est enfin dans un milieu essentiellement urbain.

Image 5

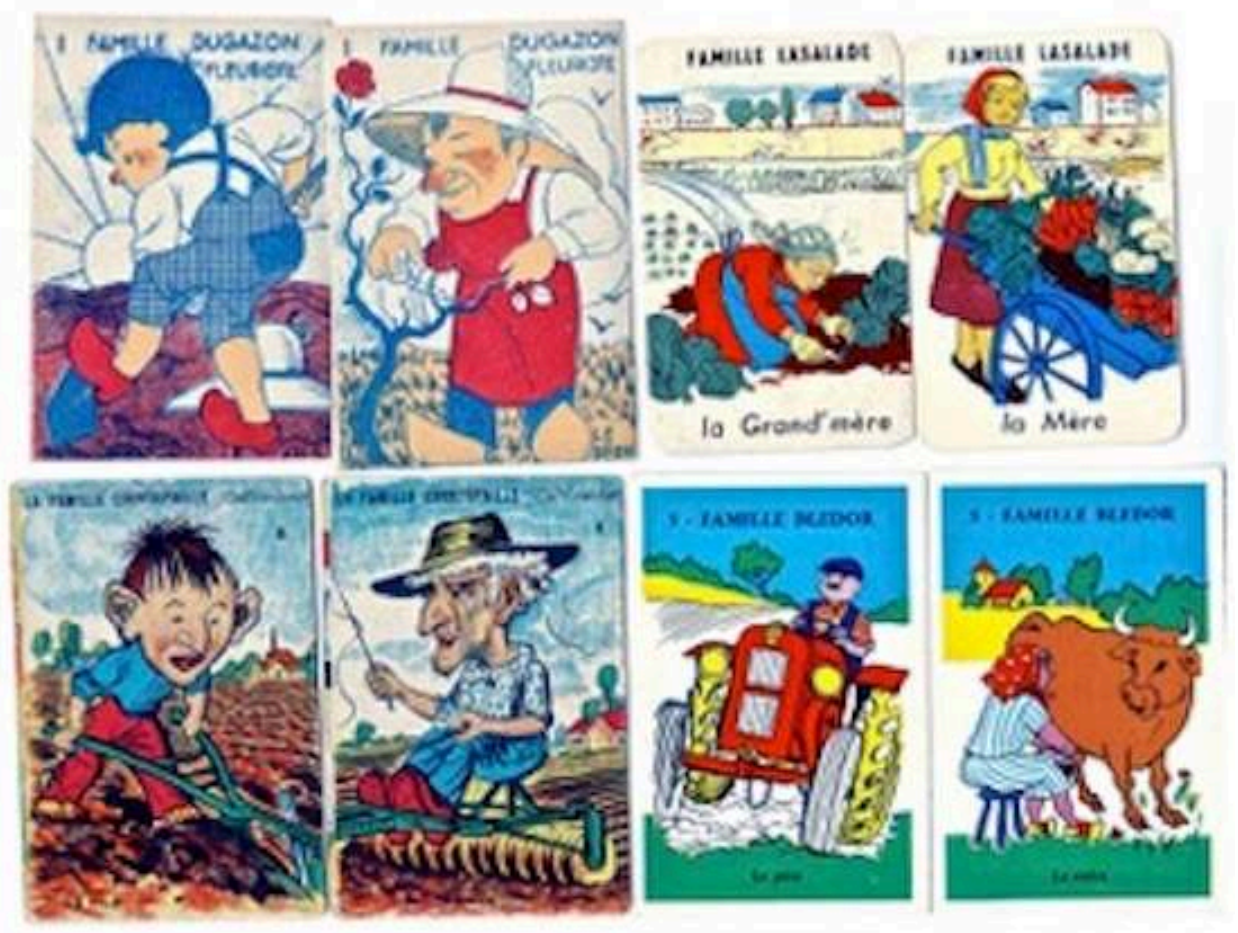

La concurrence entre les cartiers et la nécessité de renouveler les jeux conduisent à certains glissements dans les métiers, le charcutier remplaçant le boucher, le pâtissier se substituant au boulanger, puis le cuisinier prenant la place soit du pâtissier, soit du charcutier. Progressivement le jeu devient faubourien avec l'apparition du fleuriste, puis du jardinier et du maraîcher; il intègre la campagne lorsque le fleuriste ou le jardinier se fait paysan. Avec le paysan apparaît un autre pourvoyeur de nourriture, le pêcheur. L'évolution du jeu réintroduit les activités du jeu anglais des Joyeuses Familles. Le brasseur sera remplacé par le marchand de vin et les activités artisanales liées au logement réapparaissent sous les formes du menuisier, du maçon (qui peut éventuellement assurer aussi la peinture), du ramoneur. Le pharmacien remplacera le médecin. Des métiers nouveaux apparaîtront comme le garagiste ou le mécano. 


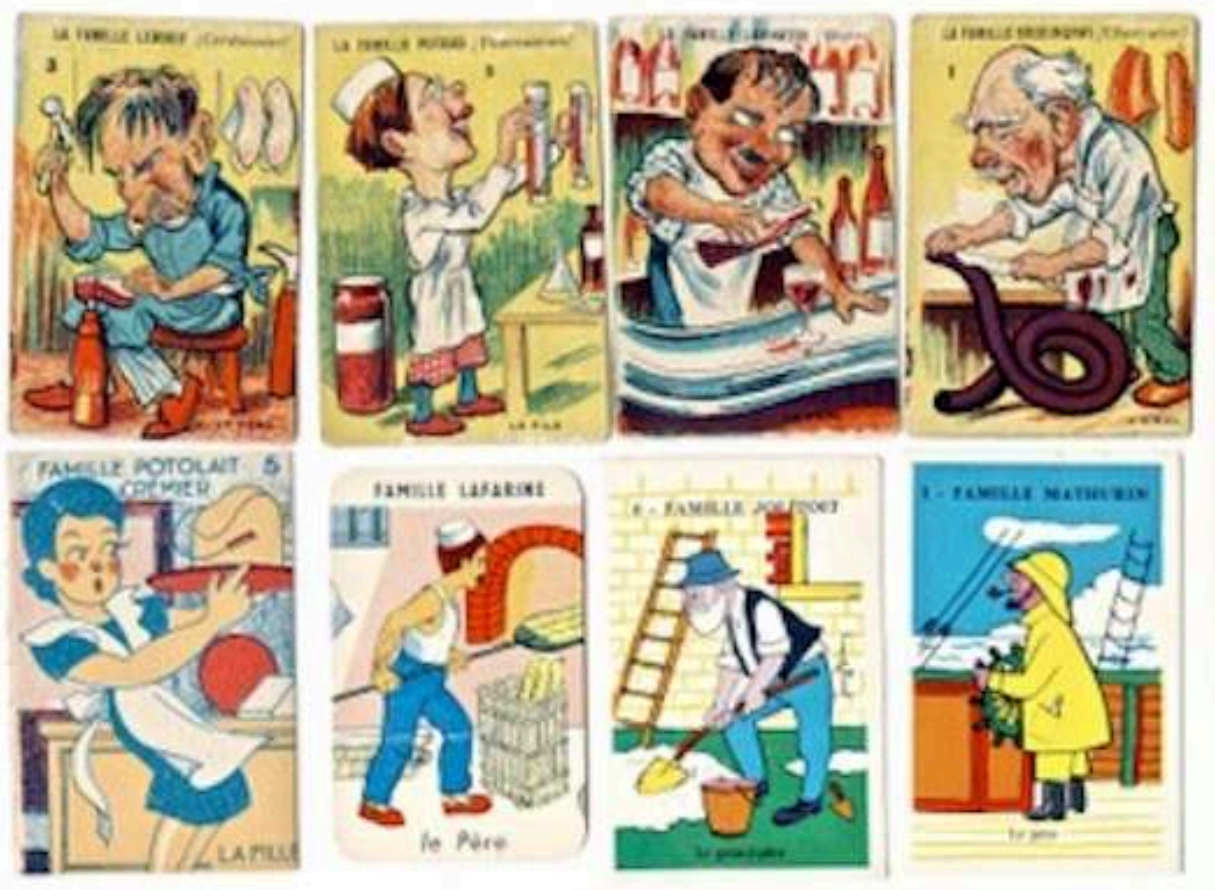

Le jeu des familles peut dans certains cas être spécialisé. C'est le cas des productions consacrées au monde du cirque et plus récemment le monde du cinéma, chaque famille représentant un des métiers possibles. Le sens de l'ensemble est quelquefois plus caché de deux façons différentes:

- Premier cas. Les métiers ou activités sont les suivants : sculpteur, acteur, peintre, poète, couturier, musicien, cinéaste. On a donc un jeu spécialisé dans les métiers artistiques. Un jeu plus récent, semble au premier abord assurer la continuité : le graveur, l'architecte, le décorateur, le dessinateur, le potier, le peintre et le sculpteur. Au premier abord, ce ne serait qu'une spécialisation de plus des arts plastiques à moins que ce ne soit, et c'est notre hypothèse, l'école des Beaux Arts.

- Second cas. Un jeu des années 1930, dont le dessin se rattache au début de l'illustré, quelque part entre Bécassine et Zig et Puce, tranche avec les jeux traditionnels. La première famille produit du lait et du beurre, la seconde du miel, la troisième de la farine, la quatrième du sucre, la cinquième des fruits, la sixième du chocolat et la dernière des œufs. Si une partie des dessins renvoie à la production et introduit l'industrialisation de la production (sucre et chocolat) ou la mécanisation de l'agriculture (lait et beurre), une large part des dessins n'a rien à voir avec le travail ou la production et renvoie plus au jeu ou à la gourmandise. Si l'on prend la liste des produits présents dans le jeu, on a tous les ingrédients nécessaires pour faire un gâteau.

Du même type mais plus subtil encore, le jeu offert par l'entreprise de crème de gruyère Graf. En suivant l'ordre alphabétique le jeu se compose de :

- Famille Ananas (production de fruits)

- Famille Cochonnaille (Charcutier)

- Famille Crevette (Poissons et Crustacés)

- Famille Lafarine (Boulanger)

- Famille Lasalade (Maraîcher) 
- Famille Picollo (Vin)

- Famille Tartinette (Fromage Graf) des tartinettes Graf. Sous-entendu, c'est un fromage qui a sa place dans un repas.

Image 7

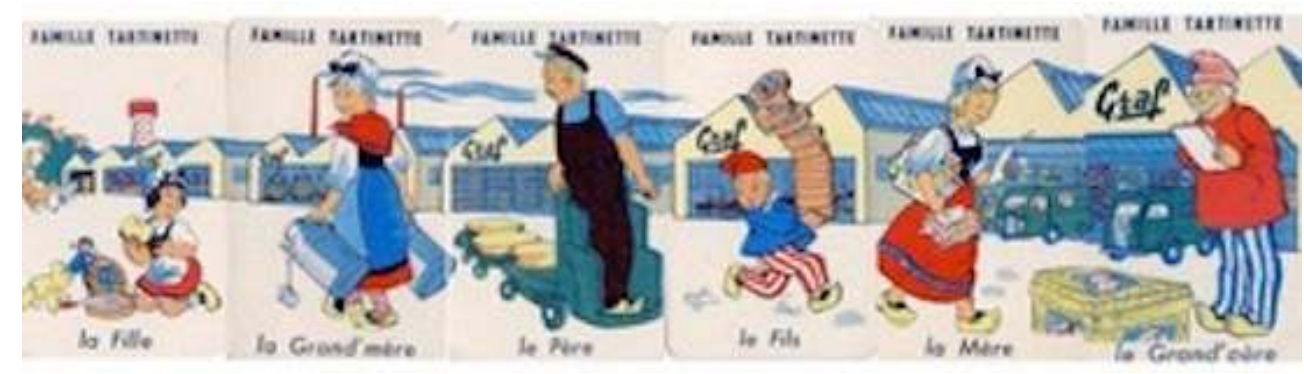

La famille Tartinette montre que Graf est une entreprise industrielle qui a gardé son caractère familial et qui n'a pas rompu avec le monde paysan (les vaches viennent à l'usine se faire traire, activité qui incombe à la grand-mère).

On voit apparaître ici la façon dont la structure du jeu limite la représentation du travail réel. La structure familiale rend difficile l'introduction du travail industriel, qui, lui, ne s'exécute pas dans le cadre de la petite production familiale. Ce n'est donc qu'une partie du monde du travail qui peut trouver place dans le jeu et la partie la plus stable dans ses pratiques. Certes on peut saisir quelquefois l'évolution technique comme chez le cordonnier-bottier. Là où le grand-père monte tout à la main, le père et la mère ont recours à la machine à coudre. C'est aussi le tracteur qui apparaît dans l'agriculture ou de nouveaux métiers qui s'adaptent à la structure familiale comme le garagiste-pompiste.

31 L'attachement de la clientèle du jeu à certaines représentations de métiers, le nombre relativement réduit de métiers utilisables (connus de tous et surtout des enfants), la nécessité de renouvellement des jeux pour continuer à vendre ce bien durable, les techniques de production pour produire à bas pris, tous ces éléments ont contribué à réduire les marges de liberté dans l'innovation et à copier des éléments des jeux anciens les plus populaires.

En même temps, les jeux des familles se détacheront progressivement des métiers pour investir le monde des objets et des personnages de dessins animés ou de bandes dessinées. On peut se demander si ce mouvement coïncide avec le recul de l'importance ou de l'attrait du travail dans la société. Enfin, les cartiers, plutôt que de créer des nouveaux jeux de métiers, jouent souvent pour l'intérêt croissant pour les objets du passé et se contentent quelquefois de réédition.

Deux jeux récents d'origine belge vont nous permettre de mesurer le chemin parcouru. Les personnages sont issus d'une bande dessinée et sont utilisés dans un ensemble de jeu des Sept Familles. Nous avons vu comment depuis l'origine, l'horticulteur est devenu maraîcher, puis paysan. Avec Marc et Julie, nous retrouverons la famille Deschamp dans la série loisirs : le père tond le gazon, le fils s'occupe de fleurs, la mère cueille des fruits et la fille donne des graines (conditionnées en boîtes) aux oiseaux, tandis que la grand-mère tricote et que le grand-père dort sous un arbre. 


\section{L'évolution technique}
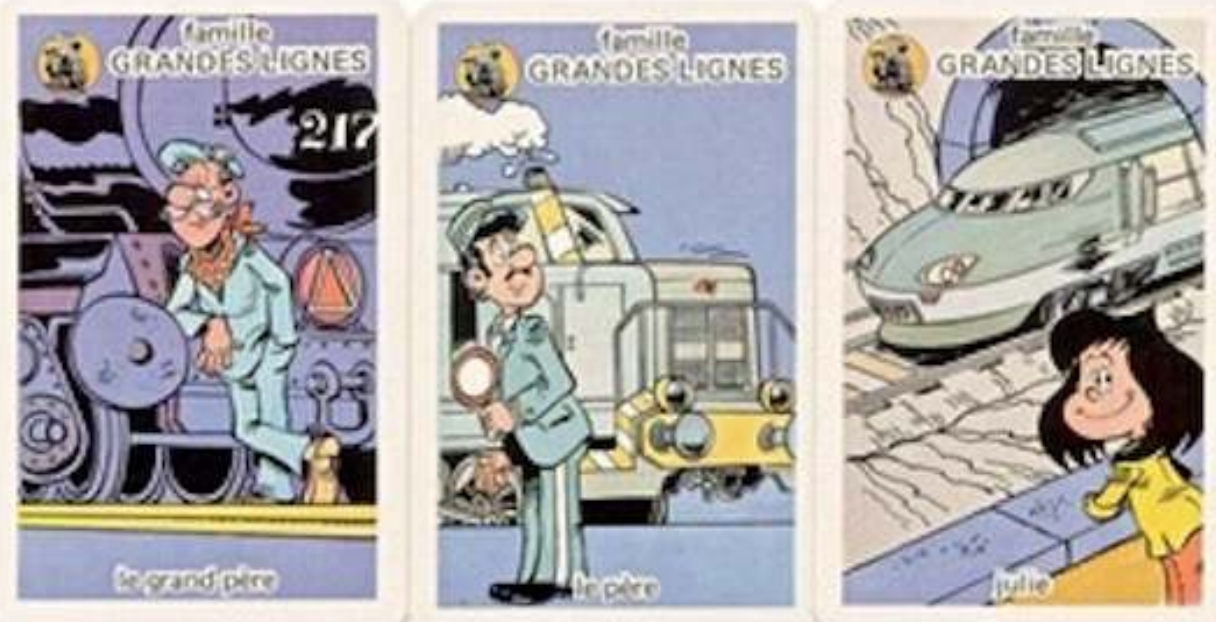

Plus significatif encore leur incursion dans les transports. Pour les chemins de fer : le grand-père est mécanicien à la traction à vapeur, la grand-mère est employée à la voie comme garde barrières; à la génération suivante, le père est devenu sédentaire employé à la gare, tandis que la mère utilise le train pour aller travailler ; Marc et Julie rêvent sur les nouvelles formes de train. Pour la navigation, le grand-père est officier de marine à l'époque des voiliers et des bateaux à roues; le père et la mère rêvent sur les paquebots ; Julie est séduite par les aéroglisseurs tandis que Marc fait du hors-bord. La bicyclette et la moto enfin, qui pour le grand-père étaient découvertes et avant-garde, puis instruments de transport ou de travail pour le père, sont devenus instruments de sport et de parade pour Marc.

On voit ainsi que le travail n'a pas totalement été évacué des jeux mais qu'il faut le chercher ailleurs que dans les jeux de métier.

\section{L'hymne au paysan}

Le jeu de tarot tel que nous le connaissons et l'utilisons aujourd'hui est une création de la fin du XIXe siècle et remplace les tarots dérivés peu ou prou des tarots de Venise (plus connus sous le nom de tarot de Marseille). Les 21 atouts (ou arcanes) de ce nouveau tarot nous présentent deux mondes tête-bêche; un univers campagnard de "gens qui peinent pour vivre » et un univers citadin « de gens qui peuvent vivre sans travailler ", pour reprendre l'exposé de Jules Payot, qui fut recteur de l'Académie d'AixMarseille, dans ses Conseils et directions pratiques aux instituteurs et institutrices au début du XXe siècle. L'univers présenté correspond tout à fait à la production chansonnière (Le credo du paysan, La chanson des blés d'or, L'angélus, etc.), à la peinture de Millet et à la mission confiée aux instituteurs et institutrices qui, tout en répandant le savoir de la République, doivent freiner l'exode rural en menant la guerre aux villes: "L'institutrice saura, elle aussi, persuader qu'à la campagne, la vie des jeunes femmes, pourvu qu'elles sachent améliorer leur existence par leur ingéniosité, est bien plus 
saine, plus libre, plus heureuse que la vie à la ville. Elle dépeindra le sort des jeunes filles qui vont comme domestiques à la ville, et qui sont mal logées, souvent mal nourries, sans affection réelle, accablées de travail. Mieux vaut la liberté, même un peu rude des champs, et le grand air et la vie au milieu des amies d'enfance ».

Image 9

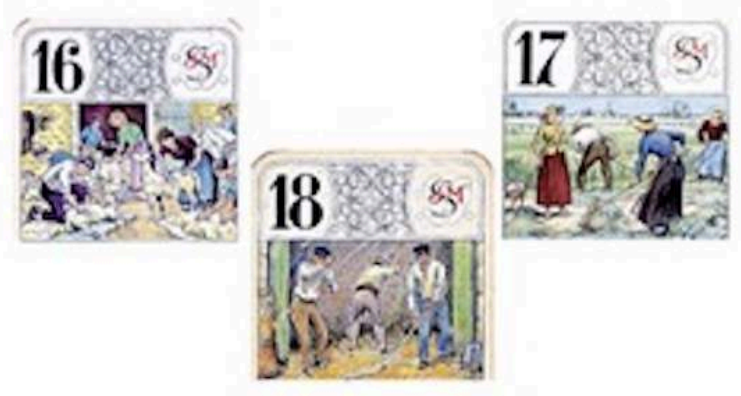

La ruche campagnarde travailleuse et productrice

La ville spectatrice et consommatrice

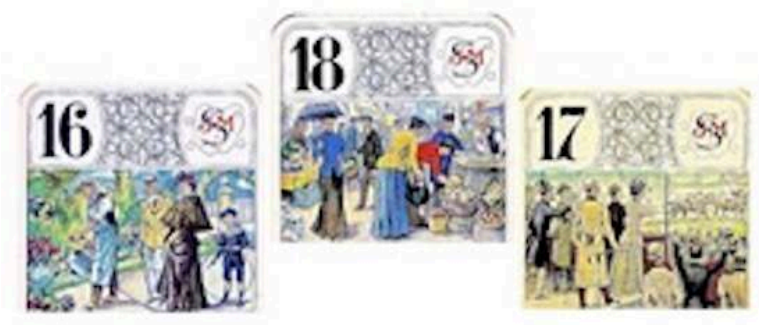

La campagne est le lieu où peut se manifester le mieux l'idéal républicain sans être masqué par la lutte des classes qui ne contribue guère à l'édification morale: «L'argent a créé des classes : les hommes se résignent si difficilement à être humains ; l'orgueil qui serait, comme on l'a dit, la plus forte preuve de l'origine simiesque de l'homme, corrompt si bien le jugement, que, sauf à la campagne où l'on estime souvent les gens d'après leurs qualités, chacun cherche à "se trier». Il faut avouer que trop souvent encore les ouvriers ne font pas ce qu'ils peuvent pour rapprocher les distances; une part importante de responsabilité leur incombe. Pourquoi tant d'entre eux affectent-ils des manières grossières, un langage incorrect et malséant, des plaisanteries ordurières, et dans leur tenue, un laisser-aller, une négligence, une malpropreté même, qui choquent?»

Plus même, la diffusion de l'idéal du travail manuel sans lequel il n'y a pas de société démocratique, est entravée par le comportement des ouvriers :

"Malheureusement les revendications bruyantes des ouvriers des villes tendent à appeler l'attention surtout sur le travail des usines: travail monotone, sans initiative de la part de l'ouvrier, devenu l'esclave de la machine, et qui tend à décourager et à abêtir celui qui y est soumis trop longtemps. Pour ces motifs, ce travail sera le dernier réhabilité : il n'est d'ailleurs heureusement le lot que d'une petite portion de la Nation. Tous les autres métiers font intervenir la personnalité de l'ouvrier, son intelligence, sa décision. Les millions d'agriculteurs surtout, ont un travail à la fois très varié et très personnel. » 
Le travail ainsi conçu ouvre la perspective du développement humain le plus riche :

« À tout bien considérer, s'ils sont instruits, le menuisier et le jardinier, par la nature même de leur travail, ont plus d'occasions de cultiver la finesse de leurs sens, de développer leur énergie et leur vigueur morale, que l'employé qui s'amollit dans une profession trop sédentaire. Le travail manuel et surtout le travail au grand air, n'est exclusif ni d'un jugement sain, ni d'une culture sérieuse de la volonté, ni d'un vif sentiment de la solidarité humaine et de la beauté des choses. »

«Sauf à la campagne", «les millions d'agriculteurs surtout ", "surtout le travail au grand air » : autant de phrases qui mettent en exergue la campagne et en font un idéal de beauté et d'humanité que la vie quotidienne fait oublier : "Combien l'existence du paysan ne serait-elle pas ennoblie s'il savait admirer la splendeur des campagnes et goûter le charme de sa vie simple, si forte, si poétique! Le cadre dans lequel se passe son existence est d'une beauté qui se renouvelle pour ainsi dire à chaque heure de la journée. C'est d'abord le lever du soleil ; c'est le chant des oiseaux, c'est l'incendie du couchant ; c'est la nuit elle-même avec son recueillement religieux inconnu à la ville». Le paysan est encore le symbole du patriotisme: "pour le paysan, cultiver intelligemment son champ, est une forme de patriotisme, puisque toute amélioration profite en fin de compte à tous ». 11 faudrait encore parler de la campagne comme lieu privilégié de la famille saine.

C'est cette valorisation de la campagne, symbolique de l'accord entre la nature et l'homme, monde de la stabilité, face à un monde urbain, instable, artificiel, qui est traduit en images dans notre jeu de tarot, deux mondes inversés qui à un moment ou un autre peuvent s'interpénétrer. Ainsi le travail, à une exception près est du côté de la campagne. À la ville, le travail représenté est celui du jardinier arrosant pelouses et plates-bandes sous le regard des citadins (atout $n^{\circ} 16$ ). Le travail est ici spectacle, tout comme le travail du paysan ou la nature sont spectacles ou loisirs pour le citadin à la campagne. Une des images (atout $n^{\circ} 14$ ) représente par exemple, une double scène de pêche : au premier plan, dans une barque on pêche au filet, tandis qu'au fond le citadin, jambe de pantalon retroussé, canne à la main, panier au côté, pêche pour son plaisir.

Le jeu ne peut toutefois être lu sans faire intervenir un second principe de lecture, celui des tarots de Marseille qu'il vient de remplacer. Sans pouvoir entrer dans le détail (ce qui dépasse le cadre de cet article), les tarots de Marseille racontent l'Apocalypse selon Jean (confondu ici avec l'évangéliste). Jean apparaît deux fois: la première sous la forme d'un fou (carte non numérotée, l'excuse des nouveaux tarots) et la seconde fois sous la forme d'un bateleur (le petit ou l'atout $\mathrm{n}^{\circ} 1$ ); trois autres évangélistes apparaissent sous la forme de la justice, de la force et de la tempérance. La carte $\mathrm{n}^{\circ} 1$ des nouveaux tarots représente la scène d'un théâtre où l'on joue la Comedia del Arte, Colombine et le fou se retrouvant du côté du monde paysan. La carte $\mathrm{n}^{\circ} 21$ des atouts du tarot de Marseille représente le monde et c'est ce thème qui est repris dans le nouveau jeu répondant à la carte $n^{\circ} 2$ qui est, selon les utilisateurs des tarots en divination, la carte qui donne sens à toutes les autres. Côté ville, des petites filles sautent à la corde et jouent au ballon, véritable apprentissage de la danse que nous retrouverons à la carte 21 dans une réunion mondaine où Pierrot réapparaît. Côté campagne, dans la tradition des bataillons scolaires et dans l'esprit de la Revanche (reconquête de l'Alsace-Lorraine), les garçons du village s'entraînent à la vie militaire ; on les retrouvera sous les drapeaux accompagnés du fou dans la carte 21 : la patrie rejoint le monde. Dans le tarot de Marseille, la carte $\mathrm{n}^{\circ} 12$ représente le pendu dont la signification est « ce qui est en haut comme ce qui est en bas ». Cela se traduit, dans le 
nouveau tarot par un bal champêtre auquel répond un bal urbain. On pourrait encore prendre la carte 9 qui représente un ermite (ou un pèlerin) avec son bâton dans le tarot de Marseille et qui est remplacé ici par un voyageur porteur lui aussi d'un bâton à l'entrée d'un village. Nous retrouverons d'autres exemples plus loin. Le choix du thème iconographique apparaît commandé par la structure des jeux antérieurs.

Le travail apparaît, pour la vie rurale dans 11 lames sur 21. C'est d'abord deux paysans armés d'une faux et d'une houe (lame 6) tandis qu'au fond un jeune conte fleurette à une jeune paysanne (contamination du tarot de Marseille ou le 6 est l'amoureux). À la lame 7, c'est l'heure du repas et les femmes ont apporté de quoi manger.

Image 10

\section{Le pâtre des montagnes pense à son avenir}
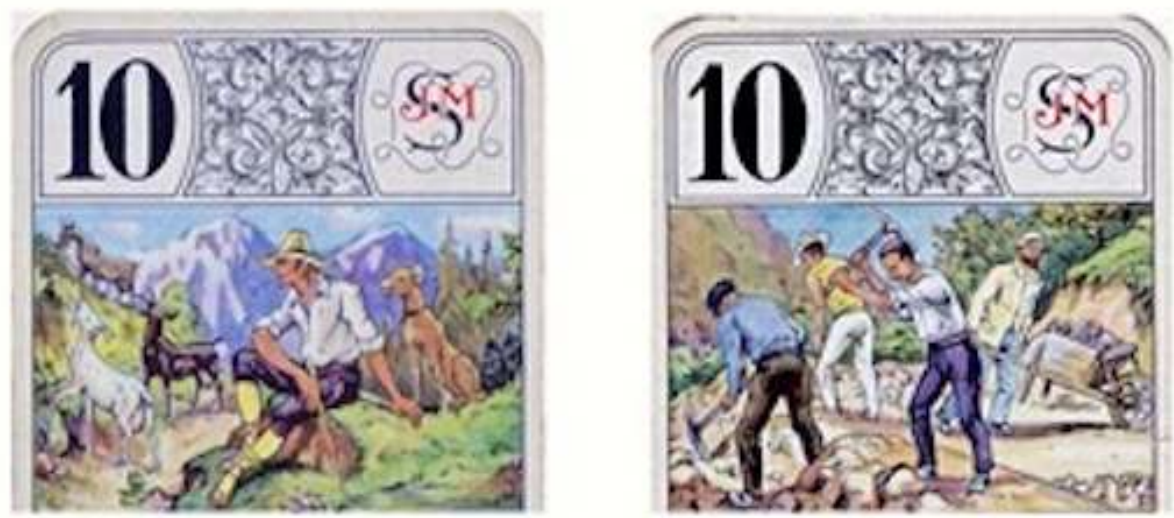

La lame 10 représente un pâtre gardant ses chèvres sur les alpages. C'est la seule lame où l'on trouve une scène de travail qui ne soit pas spectacle du côté urbain. Ce sont des cantonniers qui construisent une route, situation typique des travaux offrant au monde rural des ressources complémentaires, typique aussi des premiers travaux qui permettent de transiter du milieu paysan au monde ouvrier. Or, cette lame dans les tarots de Marseille représente la roue de fortune. On retrouve là encore la continuité entre les deux jeux. Cette lame nous ramène à nouveau aux propos déjà largement cités de Jules Payot lorsqu'i| fait ces remarques, que l'on croirait spécialement écrites pour elle :

«C'est ainsi encore que le montagnard des Alpes se familiarise si bien avec la sublimité de ses montagnes, qu'il y semble indifférent; mais vient pour celui-ci l'exil dans une grande ville, et l'étendue de la privation subie apparaît alors [...] elle deviendra ce mal meurtrier qu'on appelle mal du pays. »

Après la pêche dont nous avons déjà parlé (lame 14), vient la tonte des moutons mêlant travail des hommes et des femmes (lame 16), puis la fenaison (hommes et femmes, lame 17), le battage du blé au fléau (travail d'hommes, lame 18). Enfin à la veillée, travail de la laine (quenouille et rouet) par les femmes (lame 19). Il faudrait encore ajouter la mère s'occupant de son petit dernier tandis que sa fille apprend à tricoter (lame 4); travail des femmes encore, le tricot, la couture et la lecture des enfants (lame 5). Travail des femmes enfin, le marché (lame 13). On est donc là dans un univers moral dont 
l'iconographie dépasse largement le jeu de cartes qui fut si longtemps diabolique et le reste même ici : ce sont les citadins qui jouent aux cartes à l'intérieur tandis que les campagnards jouent aux boules en plein air dans la lame 20 qui représente le jugement dernier dans les tarots de Marseille.

Image 11

\section{Le peintre-créateur produit une image de la nature}
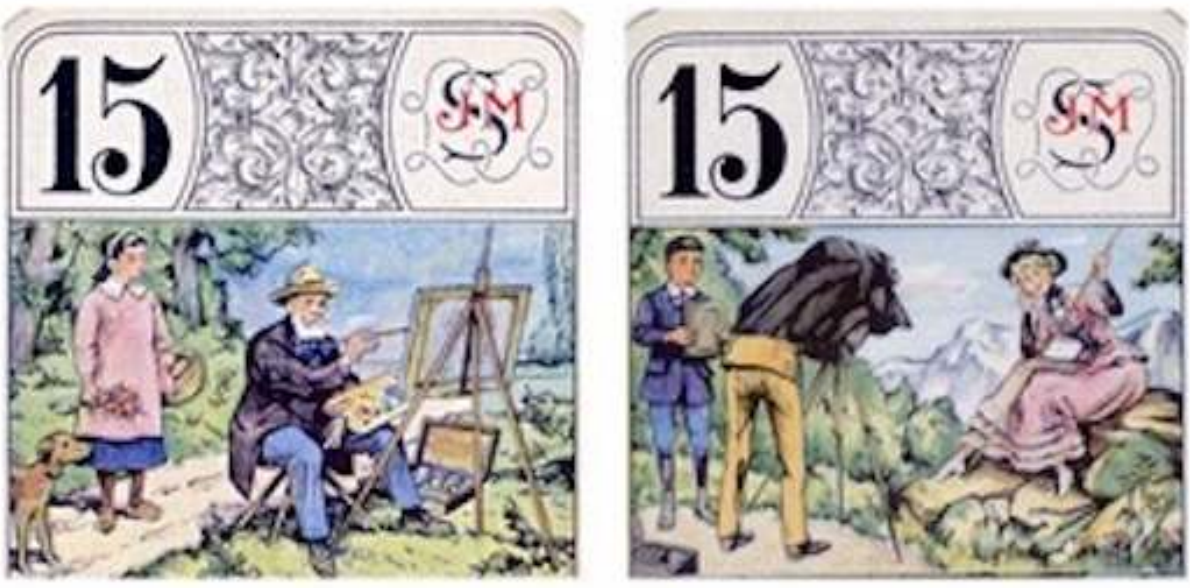

Le photographe-prédateur capte l'image de sa femme

La lame 15, celle du diable des tarots de Marseille, représentant les deux diablotins enchaînés, symbolise la formule de l'Apocalypse «ce qui fut, qui est, et qui sera » nous ramène à l'image. Dans le nouveau tarot, la lame oppose deux techniques de production d'image et deux types de représentation. Côté campagne, un peintre installé dans et face à la nature ; une petite paysanne voit la création (le travail du peintre et ce qui est sur la toile). Côté citadins, une famille en voyage à la montagne. Le père photographie la mère sur fond de montagne; l'objet de l'image a changé. Le fils tient une plaque photographique dans la main tandis que le père officie caché sous son voile noir et ne peut voir le travail de création. Mais qu'importe la création pourvu qu'on ait le produit et que celui-ci soit éducatif ajouterait Jules Payot: «On apprendra aux paysans, aux ouvriers, à détruire l'horrible imagerie qui déshonore leurs maisons. On verra les chefsd'œuvre de la peinture reproduits par la gravure ou par la photographie, occuper la place d'honneur, à côté du portrait du fils artilleur ou fantassin, et remplacer les grossières Complaintes imagées de Tropmann ou de Ravachol »?

\section{Conclusion}

L'essentiel de notre analyse a consisté à montrer la richesse des rapports entre jeu de cartes et représentations du travail et la complexité des phénomènes qui contribuent à la production matérielle de telles représentations, qu'il s'agisse de problèmes techniques, de codes iconographiques, d'utilisation des jeux ou de représentations idéologiques. Reste que nous n'avons pas abordé le second versant du problème, la façon dont ces représentations sont perçues et interprétées. Perçues lorsque les cartes 
sont regardées comme un livre d'images; perçues selon le jeu auquel on joue ; perçues quand les joueurs sont les contemporains du jeu ou au contraire lorsque les représentations appartiennent à une période historique révolue.

Retenons toutefois que la représentation s'est arrêtée à la porte de l'usine sans la franchir, laissant le champ libre à la carte postale ou à la photographie. Est-ce parce que le travail industriel n'a jamais été hautement considéré dans la société et ne pouvant de ce fait être utilisé dans une démarche éducative et morale? Est-ce parce que les conditions de travail industrielles ne pouvaient prêter à caricature ou à dérision? En ce temps, nous laisserons ces questions sans réponse.

\section{BIBLIOGRAPHIE}

Blaudez F., Jacquemin A. et Mistler J. (1961), Épinal et l'imagerie populaire, Paris Hachette.

Hoffmann D. (1972), Le monde de la carte à jouer, Éd. Leipzig.

Larmessin N. de. (1974), Les costumes grotesques, Paris, Éd. Henri Verrier.

Payot J. (1919), Aux instituteurs et institutrices. Conseils et directions pratiques, Paris Armand Colin, 10e édition.

Massin. (1978), Les cris de la ville, Paris, Gallimard.

Seguin, J.-P. (1968), Le Jeu de carte, Paris, Hermann.

\section{RÉSUMÉS}

L'auteur analyse l'image du travail diffusée dans les jeux de cartes, principalement français. Il montre que l'on a affaire à un univers très codé qui limite les possibilités d'expression à des stéréotypes. Au début du XIXe siècle, les cartes représentent les petits métiers de la rue. Dans la seconde partie du siècle, ce sont les artisans sédentaires et les boutiquiers. Ce n'est qu'à la fin du siècle que l'on verra apparaître le monde rural. Le jeu ne représentera jamais le monde industriel et intègre difficilement le progrès technique au XXe siècle. Il compare enfin les cartes d'atout nouveau jeu de tarot créé à la fin du XIXe siècle avec l'image de la ville et de la campagne diffusée dans les écoles primaires à la même époque.

This paper deal with the image of the trades spread through the playing cards, mainly french games. The cards are a strongly coded space which lead to stereotyped pictures.At the beginning of the XIX century, the cards represent the urban tramping trades. In the second part of the century they will show the sedentary craftmen and the shopkeepers. We can find the countryside activities only at the end of the century. The cards will never show pictures of the industrial work and will have difficulties to introduce the technical development. In the last part, the author compare the trumps of the new french tarot pack produced at the end of XIX century with the images of the urban and county life spread in the primary schools at the same period. 
INDEX

Mots-clés : métier, code, image, carte à jouer, histoire

Keywords : trade, code, image, playing cards, history

\section{AUTEUR}

\section{ROGER CORNU}

Roger Cornu a orienté ses travaux de recherche sur le travail et les mutations dans le secteur industriel (manutention portuaire, réparation et construction navales, mines, conserveries, ferblanteries, BTP). Iil a su mobiliser très tôt l'image photographique et l'audiovisuel comme mode de questionnement et comme instrument d'enregistrement des entretiens collectifs. sociologue, chercheur au CNRS à la retraite 